\title{
The distance function and defect energy
}

\author{
Patricio Aviles \\ Department of Mathematics and Physics, University of Oxford, Oxford, \\ U.K.; E TH. CH-8592, Zurich, Switzerland \\ Yoshikazu Giga \\ Department of Mathematics, Hokkaido University, Sapporo 060, Japan
}

(MS received 30 July 1995 )

\begin{abstract}
Several energies measuring jump discontinuities of a unit length gradient field are considered and are called defect energies. The main example is a total variation $I(\varphi)$ of the hessian of a function $\varphi$ in a domain. It is shown that the distance function is the unique minimiser of $I(\varphi)$ among all non-negative Lipschitz solutions of the eikonal equation $|\operatorname{grad} \varphi|=1$ with zero boundary data, provided that the domain is a two-dimensional convex domain. An example shows that the distance function is not a minimiser of $I$ if the domain is noncovex. This suggests that the selection mechanism by $I$ is different from that in the theory of viscosity solutions in general. It is often conjectured that the minimiser of a defect energy is a distance function if the energy is formally obtained as a singular limit of some variational problem. Our result suggests that this conjecture is very subtle even if it is true.
\end{abstract}

\section{Introduction}

It is important to measure the energy of jump discontinuities of a unit length gradient field $\nabla \varphi$ in a bounded Lipschitz domain in $\mathbb{R}^{n}$. Such problems arise in the modelling of smectic liquid crystals $[2,12]$, or of the blistering of thin films [11]. The quantity measuring the energy of the jump discontinuities, the defect of $\nabla \varphi$, is

$$
J^{\beta}(\varphi)=\int_{\Sigma}\left|\nabla \varphi^{+}-\nabla \varphi^{-}\right|^{\beta} d \mathscr{H}^{n-1},
$$

where $\beta>0$; we call it a defect energy. Here $\Sigma$ is the set of jump discontinuities of $\nabla \varphi$, and $\nabla \varphi^{ \pm}$is the trace of $\nabla \varphi$ of each side of $\Sigma ; \mathscr{H}^{n-1}$ is the $n$-1-dimensional Hausdorff measure which is the surface element when $\Sigma$ is smooth.

There may be many Lipschitz solutions of the eikonal equation

$$
|\nabla \varphi|=1 \text { in } \Omega \text { with } \varphi=0 \text { on } \partial \Omega,
$$

but the distance function

$$
d=d(x, \partial \Omega)=\inf \{|x-y| ; y \in \partial \Omega\}
$$

is the unique viscosity solution of the problem [6]. In other words, the theory of viscosity solutions selects a solution of the eikonal equation. There is a fundamental question as to whether the distance function minimises $J^{\beta}$ among all non-negative solutions of the eikonal equation. 
If the space dimension $n$ equals one, $J^{\beta}$ just measures a contact multiple of the number of jumps of $\nabla \varphi$. There is no solution of the eikonal equation having no defect satisfying the zero boundary condition. Thus, the distance function is a (unique) minimiser of $J^{\beta}$, since it has only one jump of the derivative of $\varphi$. However, for the multidimensional case, the situation is different.

In this paper, we focus on the case $\beta=1$ because of independent interest related to the total variation of the hessian

$$
I(\varphi)=\int_{\Omega}\left|\nabla^{2} \varphi\right| .
$$

This integral is closely related to $J^{1}$. Indeed, if $\varphi$ is piecewise linear, more precisely, $\nabla^{2} \varphi=0$ (as a measure) outside $\Sigma$, then

$$
I(\varphi)=\int_{\Sigma}\left|\nabla^{2} \varphi\right|=\int_{\Sigma}\left|\nabla^{+} \varphi \cdot v-\nabla \varphi^{-} \cdot v\right| d \mathscr{H}^{n-1},
$$

where $v$ is the approximate normal of $\Sigma$ [7]. Since the tangential component of $\nabla \varphi$ is approximately continuous, $\left|\nabla \varphi^{+} \cdot \nu-\nabla \varphi^{-} \cdot \nu\right|=\left|\nabla \varphi^{+}-\nabla \varphi^{-}\right|$if $|\nabla \varphi|=1$. Thus, $I(\varphi)=J^{1}(\varphi)$ for piecewise linear $\varphi$. Our principal results are as follows.

(i) The distance function is the unique minimiser of $I(\varphi)$ among all non-negative (Lipschitz) solutions of the eikonal equation $|\nabla \varphi|=1$ in $\Omega$ with $\varphi=0$ on $\partial \Omega$, provided that $\Omega$ is convex and $n=2$. The value of minimum equals $\mathscr{H}^{n-1}(\partial \Omega)$.

(ii) There is a simply connected nonconvex domain $\Omega$ in $\mathbb{R}^{2}$ such that the distance function is a minimiser of neither $J^{1}$ nor $I$.

This suggests that the selection mechanism of the ground state by $I$ or $J^{1}$ is different from that in the theory of viscosity solutions, in general.

To show (i), we first observe that

$$
|\Delta \varphi|=\left|\nabla^{2} \varphi\right|
$$

as measures if $\varphi$ solves $|\nabla \varphi|=1$ and $n=2$. This depends on the fact that $\nabla^{2} \varphi$ has rank one, which is easy to observe heuristically. Differentiating $|\nabla \varphi|=1$ implies that one of the eigenvalues of $\nabla^{2} \varphi$ always equals zero. To carry out this idea, we appeal to the theory of functions of bounded variation [7]. Note that the singular part (with respect to the Lebesgue measure) of $\nabla^{2} \varphi$ always has rank one $[1,3]$. Another key observation is

$$
\int_{\Omega}|\Delta \varphi| \geqq \int_{\Omega}-\Delta \varphi=\mathscr{H}^{n-1}(\partial \Omega),
$$

if $|\nabla \varphi|=1, \varphi \geqq 0$ in $\Omega$ with $\varphi=0$. The last equality formally follows from integration by parts and the fact that $|\nabla \varphi|$ agrees with the inward normal derivative of $\varphi$ on $\partial \Omega$. In Section 2, we state these observations in a rigorous way, allowing that $\nabla^{2} \varphi$ is a measure. If $\Omega$ is convex, the distance function $d$ is concave in $\Omega$, so that $-\Delta d \geqq 0$ in $\Omega$ (in the distribution sense). Thus

$$
\int_{\Omega}|\Delta d|=\int_{\Omega}-\Delta d=\mathscr{H}^{n-1}(\partial \Omega)
$$

so that $d$ minimises $I$ as well as $\int_{\Omega}|\Delta \varphi|$. It turns out that $d$ is a unique minimiser 
among all $\varphi,|\nabla \varphi|=1, \varphi \geqq 0$ in $\Omega$ with $\varphi=0$ on $\partial \Omega$. The inequality

$$
\int_{\Omega}|\Delta \varphi| \geqq \int_{\Omega}-\Delta \varphi
$$

is not sharp unless $\Omega$ is convex. In other words, the minimum of $I$ is strictly greater than $\mathscr{H}^{n-1}(\partial \Omega)$ (Theorem 2.8). The proof of (ii) depends on an explicit construction of the domain $\Omega$.

As a corollary of (i), we get: if $d$ is a piecewise linear, more precisely, $\nabla^{2} d=0$ outside the defect as a measure, then $d$ also minimises $J^{1}$ (among all non-negative solutions of the eikonal equations) provided that the domain is convex. Note that such $d$ exist if and only if the domain is a convex polygon as shown in Remark 2.2.

Our counterexamples are interesting for the study of minimisers of the singular perturbed variational problem

$$
E_{\varepsilon}(\varphi)=\int_{\Omega} W(\nabla \varphi)+\varepsilon^{2} \int_{\Omega}\left|\nabla^{2} \varphi\right|^{2}, \quad W(p)=\left(1-|p|^{2}\right)^{\sigma}, \quad \sigma>0,
$$

in a plane domain $\Omega$ with $\varphi=0$ on $\partial \Omega$. Since the Euler-Lagrange equation is fourth order, we are entitled to impose another boundary condition. The natural choice seems to be $\partial \varphi / \partial v=-1$, where $v$ is the unit outward normal of $\partial \Omega$. We divide $E_{\varepsilon}$ by $\varepsilon$ so that we hope that the energy has a nonzero limit as $\varepsilon \rightarrow 0$.

Formal analysis for $\sigma=2$ done in $[2,11]$ suggest that this problem has Gammalimit

$$
\begin{gathered}
\tilde{J}=2 \int_{\Sigma} \int_{-b}^{b}\left|1-\left(a^{2}+\tau^{2}\right)\right|^{\sigma / 2} d \tau d \mathscr{H}^{n-1}, \\
a=\left|(\nabla \varphi)_{\tan }\right|, \quad b=\left(1-a^{2}\right)^{1 / 2},
\end{gathered}
$$

where $(\nabla \varphi)_{\tan }$ denotes the tangential component of $\nabla \varphi^{+}$(or $\nabla \varphi^{-}$). Since $|\nabla \varphi|=1$, we see that $\tilde{J}$ is a positive constant times $J^{\sigma+1}$. This $\tilde{J}$ (or $J^{\sigma+1}$ ) is to be minimised subject to the same boundary condition as for $E_{\varepsilon}$, and the interior condition $|\nabla \varphi|=$ 1 a.e. in $\Omega$.

It is tempting to think that the minimiser $\varphi_{\varepsilon}$ of $E_{\varepsilon}$ tends to

$$
\varphi_{0}(x)=d(x, \partial \Omega)
$$

as $\varepsilon \rightarrow 0$. Similarly, it is tempting to think that this function might be a minimiser of $\tilde{J}$ (or $J^{\sigma+1}$ ). These conjectures are more or less explicit in [11] (cf. [2] for $\sigma=2$ ).

An extended version of our examples (Theorem 3.3) says that the second conjecture is false for some nonconvex domain, at least for $\sigma<\beta_{0}-1$ with some $\beta_{0}>1$ close to one. Unfortunately, in our examples $\beta_{0}$ is less than 3 , so they do not solve the original conjecture for $\sigma=2$. However, they are important because they show some possible pitfalls. In particular, they show that if these conjectures are true for $\sigma=2$, then the reasons must be subtle, since other equally reasonable-sounding statements are false.

The limiting process of $E_{\varepsilon}$ as $\varepsilon \rightarrow 0$ is not at all clear compared with the case when $\nabla \varphi$ is a scalar function. Such a convergence problem is studied in $[8,9]$ for $\nabla \varphi$ when $W$ has isolated equal minima. 


\section{Estimate of total variations of gradient field of length one}

We are concerned with the total variation of $\nabla^{2} \psi$ in a bounded two-dimensional domain $\Omega$ when $|\nabla \psi|=1, \psi \geqq 0$ on $\Omega$ and $\psi=0$ on the boundary $\partial \Omega$. Our principal result in this section is that the minimum of the total variation is attained (uniquely) at the distance function, provided that $\Omega$ is convex.

Notation 2.1. Let $\Omega$ be a bounded domain in $\mathbb{R}^{n}$ with Lipschitz boundary $\partial \Omega$. For a Lebesgue integrable function $\varphi$, i.e. $\varphi \in L^{1}(\Omega)$, let $\nabla \varphi=\left(\partial_{i} \varphi\right)_{i=1}^{n}$ and $\nabla^{2} \varphi=\left(\partial_{i} \partial_{j} \varphi\right)$ $(1 \leqq i, j \leqq n)$, a distributional gradient and hessian of $\varphi$, respectively. Let $X$ be the space of $\varphi \in L^{1}(\Omega)$ such that $\partial_{i} \varphi \in L^{1}(\Omega)(1 \leqq i \leqq n)$ and $\partial_{i} \partial_{j} \varphi$ is a finite Radon measure on $\Omega(1 \leqq i, j \leqq n)$. In other words, $\partial_{i} \varphi$ is a function of (essentially) bounded variation, i.e. $\partial_{i} \varphi \in B V(\Omega)$. Let us recall the fundamental decomposition of $\nabla^{2} \varphi$ for $\varphi \in X$; see e.g. [3]. Let $\Omega_{0}$ be the largest subset in $\Omega$ such that $\nabla^{2} \varphi$ is absolutely continuous in $\Omega_{0}$ and let $\Sigma$ be the set of jump discontinuities of $\nabla \varphi$. Then

$$
\nabla^{2} \varphi=\nabla^{2} \varphi\left\lfloor\Omega_{0}+\nabla^{2} \varphi\left\lfloor\left(\Omega-\Omega_{0}-\Sigma\right)+\nu \otimes\left(\nabla \varphi^{+}-\nabla \varphi^{-}\right) \mathscr{H}^{n-1}\lfloor\Sigma .\right.\right.
$$

Here for a set $Z$ and measure $\mu$ we associate a new measure $\mu\lfloor Z$ by

$$
(\mu\lfloor Z)(B)=\mu(Z \cap B), \quad B \subset \Omega .
$$

The vector field $v$ is the approximate unit normal of $\Sigma$ and $\nabla \varphi^{ \pm}$is the trace of $\nabla \varphi$ on $\Sigma$ in the direction of $\pm v ; \mathscr{H}^{k}$ denotes the $k$-dimensional Hausdorff measure. The first term $\left(\nabla^{2} \varphi\right)^{a b}=\nabla^{2} \varphi\left\lfloor\bar{\Omega}_{0}\right.$ is often called the absolutely continuous part of $\nabla^{2} \varphi$. We always identify $\left(\nabla^{2} \varphi\right)^{a b}$ with the corresponding locally Lebesgue integrable function in $\Omega_{0}$. The second term is often called the mild part and it lies on a nonrectifiable set $\Omega-\Omega_{0}-\Sigma$ of Lebesgue measure zero. The sum of the last two terms is called the singular part of $\nabla^{2} \varphi$. Let $Y$ be the space of $\varphi \in X$ such that $\nabla \varphi$ has no absolutely continuous part and no mild part. In other words

$$
Y=\left\{\varphi \in X ; \nabla^{2} \varphi=\varphi=v \otimes\left(\nabla \varphi^{+}-\nabla \varphi^{-}\right) \mathscr{H}^{n-1}\lfloor\Sigma\} .\right.
$$

Let $A$ be the space of $\varphi \in X$ such that $|\nabla \varphi|=1$ a.e. in $\Omega$, with $\varphi=0$ on $\partial \Omega$. We need three subclasses of $A$ :

$$
A_{+}=\{\varphi \in A ; \varphi \geqq 0 \text { in } \Omega\}, \quad A^{0}=A \cap Y, \quad A_{+}^{0}=A_{+} \cap A^{0} .
$$

We consider two integrals for $\varphi \in X$ which measure jumps of $\nabla \varphi$ :

$$
\begin{gathered}
I(\varphi)=\int_{\Omega}\left|\nabla^{2} \varphi\right|, \\
J^{\beta}(\varphi)=\int_{\Sigma}\left|\nabla \varphi^{+}-\nabla \varphi^{-}\right|^{\beta} d \mathscr{H}^{m-1} \text { for } \beta>0 .
\end{gathered}
$$

Since $\nabla^{2} \varphi$ is a finite Radon measure, in the representation

$$
\int_{\Omega}\left|\nabla^{2} \varphi\right|=\sup \left\{\sum_{1 \leqq i, j \leqq n} \int_{\Omega} \theta^{i j} \partial_{i} \partial_{j} \varphi ; \sum_{i, j}\left|\theta_{i j}\right|^{2} \leqq 1\right\}
$$

the test function $\theta_{i j}$ is allowed to be $\theta_{i j} \in C^{1}(\bar{\Omega})$, not necessarily compactly supported. 
REMARK 2.2. The set $A_{+}^{0}$ and even $A^{0}$ may be empty. In fact, $A_{+}^{0}$ (and $A^{0}$ ) is empty if $\partial \Omega$ has a 'curved' part. Conversely, $A_{+}^{0}$ is nonempty if $\Omega$ is a polygon. The proof is by the induction of numbers of vertices of $\Omega$. If $\Omega$ is a triangle, the distance function $d$ is certainly piecewise linear. If $\Omega$ is a polygon of $m(>3)$ vertices, we set

$$
\begin{aligned}
\rho(x, \partial \Omega)= & \min \{d(x, L(S)) ; L(S) \text { is a straight line containing an edge } S \text { of } \partial \Omega\}, \\
d_{*}= & \inf \left\{\rho(x, \partial \Omega) ; \text { there are at least three edges } S_{1}, S_{2}, S_{3} \text { of } \partial \Omega\right. \\
& \text { such that } \left.\rho(x, \partial \Omega)=d\left(x, L\left(S_{i}\right)\right), i=1,2,3 \text { and } x \in \Omega\right\} .
\end{aligned}
$$

It is easy to see that $\rho$ is piecewise linear in

$$
\Omega_{*}=\left\{x \in \Omega, d(x)<d_{*}\right\} .
$$

By the choice of $d_{*}$, the set $K=\Omega-\Omega_{*}$ is a closed polygon with at most $m-1$ vertices; it may have no interior so that $K$ is a set of points or segments. Let $\Omega^{\prime}$ be the interior of $K$ so that it is a polygon with at most $m-1$ vertices. By the induction, the function $\rho\left(x, \partial \Omega^{\prime}\right)$ is piecewise linear in $\Omega^{\prime}$. Since

$$
\rho(x, \partial \Omega)=\rho\left(x, \partial \Omega^{\prime}\right)+\mathrm{d}_{*} \quad \text { in } \mathbf{\Omega}^{\prime},
$$

and $\rho=\rho(x, \partial \Omega)$ is piecewise linear in $\Omega_{*}$, we see that $\rho$ is piecewise linear in $\Omega$. This shows that $\rho \in A_{+}^{0}$ is nonempty.

Note that $\rho$ is the distance function $d$ if and only if $\Omega$ is a convex polygon. If $\Omega$ is nonconvex, $d$ is not piecewise linear, so $d \in A_{+}^{0}$ if and only if $\Omega$ is a convex polygon.

We conclude this remark by pointing out that there is a domain $\Omega$ whose boundary is piecewise linear with infinite vertices such that $\rho \in A_{+}^{0}$. For example, if we consider

$$
\begin{gathered}
\Omega=\{(x, y) ;|x|<1,1>y>h(x)\}, \\
h(x)= \begin{cases}\frac{1}{2^{l+1}}-\left|x-\frac{3}{2^{l+1}}\right| & \frac{1}{2^{l}}<x \leqq \frac{1}{2^{l-1}}, \quad l=1,2, \ldots \\
0 & x \leqq 0,\end{cases}
\end{gathered}
$$

then $\rho \in A_{+}^{0}$. See Figure 2.1 .

Comparison Lemma of hessian AND Laplacian measure 2.3. Assume that $n=2$.

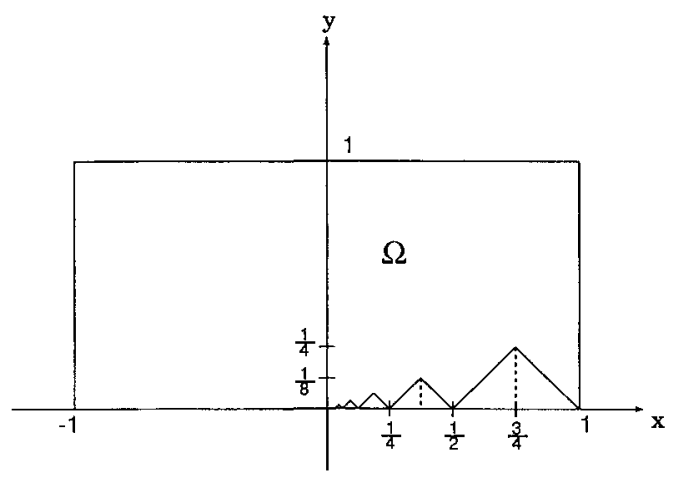

Figure 2.1. 
Then for $\varphi \in A$,

$$
|\Delta \varphi|=\left|\nabla^{2} \varphi\right| \quad \text { (as measures). }
$$

This is formally true since $\nabla^{2} \varphi$ is rank one. Indeed, differentiating $|\nabla \varphi|^{2}=1$ yields

$$
\sum_{j=1}^{n}\left(\partial_{i} \partial_{j} \varphi\right) \partial_{j} \varphi=0
$$

We shall justify this observation for general hessian measure $\nabla^{2} \varphi$ of $\varphi \in A$. We say that for $\varphi \in X$, the rank of matrix of the Radon-Nikodym derivative

$$
F(x)=\lim _{r \downarrow 0} \nabla^{2} \varphi\left(B_{r}(x)\right) /\left|\nabla^{2} \varphi\right|\left(B_{r}(x)\right)
$$

is the rank of $\nabla^{2} \varphi$, where $B_{r}(x)$ denotes the closed ball of radius $r$ centred at $x \in \Omega$. The rank of $\nabla \varphi$ is defined for $\left|\nabla^{2} \varphi\right|$-almost every point $x$ of $\Omega$. Since $\nabla^{2} \varphi$ is absolutely continuous with respect to $\left|\nabla^{2} \varphi\right|$,

$$
|\nabla \varphi|(Z)=\int_{Z}|\operatorname{trace} F| d \mu, \quad\left|\nabla^{2} \varphi\right|(Z)=\int_{Z}|F| d \mu,
$$

with $\mu=\left|\nabla^{2} \varphi\right|$, where $|F|$ is the Hilbert-Schmidt norm of $F$, i.e. $|F|^{2}=\Sigma_{i j}\left|F_{i j}\right|^{2}$. If $F$ is rank one, then

$$
\mid \text { trace } F|=| F \mid \text {, }
$$

so that $|\Delta \varphi|=\left|\nabla^{2} \varphi\right|$. Lemma 2.3 rigorously follows from the following two lemmas. LEMMA 2.4 [1]. If $\varphi \in X$, then the rank of the singular part of $\nabla^{2} \varphi\left(i . e . \nabla^{2} \varphi-\left(\nabla^{2} \varphi\right)^{a b}\right)$ is one.

This is clear if $\varphi \in Y$ because of the representation of $\nabla^{2} \varphi$. Such a property was proved for an important subset of the singular part by the present authors in [3] and conjectured there for all the singular part. This difficult problem was solved by Alberti [1]. We do not need to assume $|\nabla \varphi|=1$.

LemMa 2.5. If $\varphi \in A$, then the rank of the absolutely continuous part $\left(\nabla^{2} \varphi\right)^{a b}$ is less than or equal to $n-1$.

Proof. For $\varphi \in X$ and $j, 1 \leqq j \leqq n$, there is a representative of $u=\nabla \varphi\left(\mathscr{L}^{n}\right.$-a.e. $)$ so that the pointwise derivative $\partial u / \partial x_{j}$ exists $\mathscr{L}^{n}$-a.e. and

$$
\frac{\partial u}{\partial x_{j}}(x)=\left(\partial_{j} \nabla \varphi\right)^{a b}(x) \Omega_{0} \quad\left(\mathscr{L}^{n} \text {-a.e. }\right) \quad(1 \leqq j \leqq n),
$$

where $\mathscr{L}^{n}$ is the Lebesgue measure; see [4]. Note that the choice of $u$ may depend on $j$. Differentiating $|\nabla \varphi|^{2}=1$ in the $j$-th direction yields

$$
\begin{aligned}
0 & =2 \sum_{i=1}^{n} \frac{\partial u_{i}(x)}{\partial x_{j}} \cdot u_{i}(x)=2 \sum_{i=1}^{n}\left(\partial_{j}\left(\partial_{i} \varphi\right)\right)^{a b}(x) u_{i}(x) \\
& =2 \sum_{i=1}^{n}\left(\partial_{j}\left(\partial_{i} \varphi\right)(x)\right)^{a b}\left(\partial_{i} \varphi\right)(x) \text { for } \mathscr{L}^{n} \text {-a.e. } x \in \Omega_{0},
\end{aligned}
$$

where $u=\left(u_{i}\right)_{i=1}^{n}$. Since $|\nabla \varphi| \neq 0$ for a.e. $x$, this implies that $\left(\nabla^{2} \varphi\right)^{a b}$ has a kernel for a.e. $x$ so that the rank of $\left(\nabla^{2} \varphi\right)^{a b}$ is less than or equal to $n-1$. 
Key Lemma 2.6. For $\varphi \in X$, assume that $|\nabla \varphi|=1$ in $\Omega$ ( $\mathscr{L}^{n}$-a.e.) and $\varphi \geqq 0$ in $\Omega$ with $\varphi=0$ on $\partial \Omega$, i.e. $\varphi \in A_{+}$. Then

$$
(-\Delta \varphi)(\Omega)=\int_{\Omega}-\Delta \varphi \geqq \mathscr{H}^{n-1}(\partial \Omega) .
$$

If $\varphi(x)=\operatorname{dist}(x, \partial \Omega)$ near $\partial \Omega$, then the equality holds.

Proof. This is easy if $\varphi$ is regular so that $\varphi$ is a distance function $d(x, \partial \Omega)$ near $\partial \Omega$. Indeed, integrating by parts yields

$$
\int_{\Omega}(-\Delta \varphi) d \mathscr{L}^{n}=-\int_{\partial \Omega} \frac{\partial \varphi}{\partial \nu} d \mathscr{H}^{n-1}
$$

where $v$ is the unit outward normal of $\partial \Omega$. Since $\varphi=0$ on $\partial \Omega$ and $|\Delta \varphi|=1$ so that $v=-\nabla \varphi /|\nabla \varphi|$, the derivative $-\partial \varphi / \partial v=1$. Thus, the equality

$$
\int_{\Omega}(-\Delta \varphi) d \mathscr{L}^{n}=\mathscr{H}^{n-1}(\partial \Omega)
$$

is proved.

(1) For $\varphi \in X$, set

$$
E_{\alpha}=\{x \in \Omega ; \varphi(x) \geqq \alpha\}, \quad \alpha \geqq 0 .
$$

Since $\varphi \geqq 0$ near $\partial \Omega, \cup_{\alpha>0} E_{\alpha}=\Omega$ and $E_{\alpha}$ is decreasing in $\alpha$. Since $\varphi$ is continuous, $E_{\alpha}$ is closed for small $\alpha \geqq 0$. Since $\Delta \varphi$ is a finite Radon measure in $\Omega$, we obtain

$$
\int_{\Omega}(-\Delta \varphi)=\lim _{\alpha \downarrow 0} \int_{E_{\alpha}}(-\Delta \varphi)
$$

In particular,

$$
\int_{\Omega}(-\Delta \varphi)=\lim _{\varepsilon \downarrow 0} \frac{1}{\varepsilon} \int_{0}^{\varepsilon} d \alpha \int_{E_{\alpha}}(-\Delta \varphi) .
$$

(2) We shall prove the identity

$$
\int_{0}^{\varepsilon} d \alpha \int_{E_{\alpha}}(-\Delta \varphi)=\mathscr{L}^{n}\left(\Omega-E_{\varepsilon}\right) \text { for small } \varepsilon>0
$$

Let $\psi \in C^{2}(\Omega)$ with bounded gradient in $\Omega$. Since $\varphi$ is Lipschitz near $\partial \Omega$, the co-area formula $[7,13]$ yields

$$
\int_{\Omega-E_{\varepsilon}} \nabla \psi \cdot \nabla \varphi d \mathscr{L}^{n}=\int_{0}^{\varepsilon} d \alpha \int_{L_{\alpha}}\left(\nabla \psi, \frac{\nabla \varphi}{|\nabla \varphi|}\right) d \mathscr{H}^{n-1} \quad \text { with } L_{\alpha}=\{x \in \Omega ; \varphi(x)=\alpha\} .
$$

Since $\varphi$ is differentiable and $|\nabla \varphi(x)|=1$ for $\mathscr{L}^{n}$-a.e. $x$ (near $\partial \Omega$ ), Fubini's Theorem, for small $\left(\mathscr{L}^{1}\right.$-)a.e. $\alpha>0$,

$$
\left|\nabla \varphi\left(x_{0}\right)\right|=1 \text { for } \mathscr{H}^{n-1} \text {-a.e. } x_{0} \text { of } L_{\alpha} .
$$

For such $\alpha>0$, we may assume that the level set $L_{\alpha}$ is countably $n-1$ rectifiable, so that at $x_{0}$ the approximate outer unit normal $v_{\alpha}\left(x_{0}\right)=-\nabla \varphi\left(x_{0}\right)$. We may also assume 
that $E_{\alpha}$ is a set of finite parameter, so that the gradient $\nabla \chi_{E_{\alpha}}$ of the characteristic function $\chi_{E_{\alpha}}$ of $E_{\alpha}$ is a finite Radon measure and that

$$
\nabla \chi_{E_{\alpha}}=-v_{\alpha} \mathscr{H}^{n-1}\left\lfloor L_{\alpha} .\right.
$$

For these properties, the reader is referred to the monographs $[7,13]$. For the aboveselected $\alpha>0$, we observe that

$$
\int_{L_{\alpha}}\left(\nabla \psi \cdot \frac{\nabla \varphi}{|\nabla \varphi|}\right) d \mathscr{H}^{n-1}=\int_{\Omega} \nabla \psi \cdot \nabla \chi_{E_{\alpha}}=\int_{E_{\alpha}}(-\Delta \psi) d \mathscr{L}^{n}
$$

by integration by parts. This together with (2.2) yields

$$
\int_{\mathbf{\Omega}-E_{\varepsilon}} \nabla \psi \cdot \nabla \varphi d \mathscr{L}^{n}=\int_{0}^{\varepsilon} d \alpha \int_{E_{\alpha}}(-\Delta \psi) d \mathscr{L}^{n}
$$

We would like to take $\psi=\varphi$. Since $\varphi$ is not $C^{2}$, we need to approximate. Mollifying $\varphi$ by a standard approximation as in $[7,1.17]$ we see that there is a sequence $\psi_{j} \in C^{2}(\Omega)$ such that

$$
\begin{gathered}
\lim _{j \rightarrow \infty} \int_{\Omega}\left|\nabla \psi_{j}-\nabla \varphi\right| d \mathscr{L}^{n}=0, \quad \sup _{j \geqq 1} \sup _{\Omega}\left|\nabla \psi_{j}\right|<\infty, \\
\lim _{j \rightarrow \infty} \int_{\Omega}\left|\Delta \psi_{j}\right| d \mathscr{L}^{n}=\int_{\Omega}|\Delta \varphi|,
\end{gathered}
$$

since $|\nabla \varphi|$ is bounded. In particular, $\nabla \psi_{j} \rightarrow \nabla \varphi$ for $\mathscr{L}^{n}$-a.e. $x$ by taking a subsequence if necessary. Moreover,

$$
\left|\Delta \psi_{j}\right| \rightarrow|\Delta \varphi|, \quad \Delta \psi_{j} \rightarrow \Delta \varphi \quad \text { weakly as measures. }
$$

We shall prove that $\int_{E_{\alpha}}(-\Delta \varphi)$ is approximated by $\int_{E_{\alpha}}\left(-\Delta \varphi_{j}\right) d \mathscr{L}^{n}$. Since $-\Delta \varphi$ is a non-negative finite Radon measure and since $L_{\alpha_{1}}$ and $L_{\alpha_{2}}$ is disjoint for $\alpha_{1} \neq \alpha_{2}$, we see

$$
(-\Delta \varphi)\left(L_{\alpha^{\prime}}\right)=0
$$

except at most countably many values of $\alpha^{\prime}>0$. For the above-selected $\alpha$,

$$
\mathscr{H}^{n-1}\left(\partial E_{\alpha}-L_{\alpha}\right)=0 \text {, }
$$

since $E_{\alpha}$ is a set of finite perimeter [7].

Since $-\Delta \varphi$ is absolutely continuous with respect to $\mathscr{H}^{n-1}[7,13]$, we see

$$
(-\Delta \varphi)\left(\partial E_{\alpha}-L_{\alpha}\right)=0 \text {. }
$$

Since $E_{\alpha}$ is closed so that $\partial E_{\alpha}$ contains $L_{\alpha}$, we may assume

$$
(-\Delta \varphi)\left(\partial E_{\alpha}\right)=\int_{\partial E_{\alpha}}(-\Delta \varphi)=0
$$

for the above-selected $\alpha$ by excluding the values of $\alpha^{\prime}$ with $(-\Delta \varphi)\left(L_{\alpha^{\prime}}\right)>0$. We now apply [7, Appendix A1] to get

$$
\lim _{j \rightarrow \infty} \int_{E_{\alpha}}\left(-\Delta \psi_{j}\right) d \mathscr{L}^{n}=\int_{E_{\alpha}}(-\Delta \varphi)
$$


Since $\sup _{\Omega}\left|\nabla \psi_{j}\right|$ is bounded and $\nabla \psi_{j} \rightarrow \nabla \psi$ a.e., we now obtain

$$
\lim _{j \rightarrow \infty} \int_{\Omega-E_{\varepsilon}}\left(\nabla \psi_{j} \cdot \nabla \varphi\right) d \mathscr{L}^{n}=\int_{\Omega-E_{\varepsilon}}|\nabla \varphi|^{2} d \mathscr{L}^{n}=\mathscr{L}^{n}\left(\Omega-E_{\varepsilon}\right) .
$$

Since

$$
\sup _{\alpha, j} \int_{E_{\alpha}}\left|\Delta \psi_{j}\right|
$$

is finite, the Lebesgue Convergence Theorem yields

$$
\begin{aligned}
\lim _{j \rightarrow \infty} \int_{0}^{\varepsilon} \mathrm{d} \alpha \int_{E_{\alpha}}\left(-\Delta \psi_{j}\right) d \mathscr{L}^{n} & =\int_{0}^{\varepsilon} d \alpha \lim _{j \rightarrow \infty} \int_{E_{\alpha}}\left(-\Delta \psi_{j}\right) d \mathscr{L}^{n} \\
& =\int_{0}^{\varepsilon} d \alpha \int_{E_{\alpha}}(-\Delta \varphi) .
\end{aligned}
$$

Plugging $\psi=\psi_{j}$ into (2.3) and letting $j \rightarrow \infty$ now yields (2.1).

(3) Since $|\nabla \varphi|=1$ in $\Omega$, the set

$$
\Omega-E_{\varepsilon}=\{x \in \Omega ; 0 \leqq \varphi(x)<\varepsilon\}
$$

includes

$$
F_{\varepsilon}=\{x \in \Omega ; \operatorname{dist}(x, \partial \Omega)<\varepsilon\} .
$$

Since $\partial \Omega$ is Lipschitz, we see that

$$
\lim _{\varepsilon \downarrow 0} \mathscr{L}^{n}\left(F_{\varepsilon}\right) / \varepsilon=\mathscr{H}^{n-1}(\partial \Omega)
$$

By steps 1 and 2,

$$
\int_{\Omega}(-\Delta \varphi)=\lim _{\varepsilon \downarrow 0} \mathscr{L}^{n}\left(\Omega-\mathrm{E}_{\varepsilon}\right) / \varepsilon \geqq \lim _{\varepsilon \downarrow 0} \mathscr{L}^{n}\left(F_{\varepsilon}\right) / \varepsilon=\mathscr{H}^{n-1}(\partial \Omega) .
$$

The equality holds when $\varphi(x)=d(x, \partial \Omega)$ near $\partial \Omega$.

THEOREM 2.7 (on total variation of the Laplacian). Let $\Omega$ be a bounded domain in $\mathbb{R}^{n}$ with Lipschitz boundary.

(i) $\int_{\Omega}|\Delta \varphi| \geqq \mathscr{H}^{n-1}(\partial \Omega)$ for all $\varphi \in A_{+}$.

(ii) If $\Omega$ is convex, the minimum of $\int_{\Omega}|\Delta \varphi|$ over $A_{+}$is attained at $\varphi_{0}(x)=\operatorname{dist}(x, \partial \Omega)$ and the minimal value is $\mathscr{H}^{n-1}(\partial \Omega)$.

Proof. (i) This is a direct consequence of Lemma 2.6.

(ii) If $\Omega$ is convex, then $\varphi_{0}$ is a concave function in $\Omega$. This is easy; a similar result is proved in [5, p. 53, Lemma]. In particular, $-\Delta \varphi=|\Delta \varphi|$ as a measure. Thus, Lemma 2.6 yields

$$
-\int_{\Omega} \Delta \varphi_{0}=\mathscr{H}^{n-1}(\partial \Omega)
$$

so $\varphi_{0}$ is a minimiser of $I$ over $A_{+}$.

THEOREM 2.8 (on total variation of the hessian). Let $\Omega$ be a bounded domain in $\mathbb{R}^{n}$ with Lipschitz boundary. 
(i) $I(\varphi)=\int_{\Omega}\left|\nabla^{2} \varphi\right| d x \geqq \mathscr{H}^{n-1}(\partial \Omega)$ for all $\varphi \in A_{+}$with $n \leqq 2$, or for all $\varphi \in A_{+}^{0}$ for arbitrary $n$.

(ii) If $\Omega$ is convex, the minimum of I over $A_{+}$(with $n \leqq 2$ ) or over $A_{+}^{0}$ is uniquely attained at $\varphi_{0}(x)=\operatorname{dist}(x, \partial \Omega)$ and

$$
I\left(\varphi_{0}\right)=\mathscr{H}^{n-1}(\partial \Omega)
$$

(iii) If $\Omega$ is not convex, $I(\varphi)>\mathscr{H}^{n-1}(\partial \Omega)$ for all $\varphi \in A_{+}$(with $n \leqq 2$ ) or for all $\varphi \in A_{+}^{0}$.

Proof. (i) By Lemma 2.3, we see

$$
|\Delta \varphi|=\left|\nabla^{2} \varphi\right| \quad \text { for } \varphi \in A \quad \text { with } n=2 ;
$$

this equality is also true for $\varphi \in A^{0}=A \cap Y$ or for $\varphi \in A$ with $n=1$, since $\nabla^{2} \varphi$ is rank one. Theorem 2.7(i) now yields (i).

(ii) Since $|\Delta \varphi|=\left|\nabla^{2} \varphi\right|$, this follows from Theorem 2.7(ii) except for the uniqueness of the minimiser. Suppose that $I(\varphi)=\mathscr{H}^{n-1}(\partial \Omega)$; then

$$
\int_{\Omega}\left|\nabla^{2} \varphi\right|=\int_{\Omega}|\Delta \varphi|=\int_{\Omega}-\Delta \varphi .
$$

In particular $-\Delta \varphi \geqq 0$ as a measure. Since $\nabla^{2} \varphi$ is rank one, this means that $\varphi$ is concave. A concave function $\varphi$ in $A_{+}$is a unique viscosity solution of $|\nabla \varphi|=1$ with $\varphi=0$ on $\partial \Omega$, see e.g. [10], so $\varphi$ is the distance function $\varphi_{0}$.

(iii) If there is $\varphi$ such that $I(\varphi)=\mathscr{H}^{n-1}(\partial \Omega)$, we see $\varphi$ is a concave distance function $\varphi_{0}$ as in (ii). However, such a distance function $\varphi_{0}$ is concave in $\Omega$ if and only if $\Omega$ is convex. Thus, the strict inequality holds for a nonconvex domain.

REMARK 2.9. The uniqueness of minimisers of Theorem 2.7 (ii) would be true if $\varphi \in A_{+}$satisfying $-\Delta \varphi \geqq 0$ would be a viscosity solution of $|\nabla \varphi|=1$ (without assuming that $\varphi$ is concave). However, we do not attempt to discuss this problem here.

\section{Counterexample}

We shall construct a simply connected but nonconvex domain in $\Omega$ in $\mathbb{R}^{2}$ such that the distance function minimises neither $J^{1}$ nor $I$ among the class $A, A_{+}, A^{0}, A_{+}^{0}$ defined at the beginning of Section 2 (Notation 2.1).

\section{Choice of domain}

For a positive constant $l$, let $D_{l}$ be a square of the form

$$
D_{l}=\{(x, y) ;|y|<l, 0<x<2 l\} \text {. }
$$

Let $D$ be a unit square of the form

$$
D=\{(x, y) ;|y|<1 / 2,-1<x<0\} .
$$

Let $\Omega_{l}$ be the interior of the union of $\bar{D}$ and $\bar{D}_{l}$, where the bar denotes the closure. We shall always assume that $l>1 / 2$. Clearly, $\Omega$ is a bounded simply connected but nonconvex domain. 


\section{Defects}

We consider three solutions of the eikonal equation

$$
|\nabla \varphi|=1 \text { in } \Omega_{l} \text { with } \varphi=0 \text { on } \partial \Omega_{l}
$$

of the form:

$$
\begin{aligned}
& \varphi_{0}(x)=\operatorname{dist}\left(x, \partial \Omega_{l}\right), \\
& \varphi_{1}(x)= \begin{cases}-\operatorname{dist}(x, \partial D) & \text { for } x \in \bar{D} \\
\operatorname{dist}\left(x, \partial D_{l}\right) & \text { for } x \in \bar{D}_{l},\end{cases} \\
& \varphi_{2}(x)= \begin{cases}\operatorname{dist}(x, \partial D) & \text { for } x \in \bar{D} \\
\operatorname{dist}\left(x, \partial D_{l}\right) & \text { for } x \in \bar{D}_{l} .\end{cases}
\end{aligned}
$$

The set of jump discontinuities of $\nabla \varphi_{1}$ consists of

$$
\begin{array}{ll}
L_{1}: y=l-x, \quad 0<x<2 l, & L_{2}: y=x-l, \quad 0<x<2 l, \\
L_{3}: y=-\frac{1}{2}-x, \quad-1<x<0, & L_{4}: y=\frac{1}{2}+x, \quad-1<x<0 .
\end{array}
$$

The magnitude of jumps

$$
j=\left|\nabla \varphi^{+}-\nabla \varphi^{-}\right|
$$

on each defect is $\sqrt{2}$. For later convenience, we decompose defects of $\varphi_{1}$ :

$$
\begin{array}{ll}
\Sigma_{1}^{+}=L_{3} \cap R_{1}, & \Sigma_{1}^{-}=L_{4} \cap R_{1}, \\
S_{1}^{+}=L_{4} \cap R_{2}, & S_{1}^{-}=L_{3} \cap R_{2}, \\
\Sigma_{2}^{+}=L_{1} \cap R_{3}, & \Sigma_{2}^{-}=L_{2} \cap R_{3}, \\
S_{2}^{+}=L_{1} \cap R_{4}, & S_{2}^{-}=L_{2} \cap R_{4}, \\
\Sigma_{3}^{+}=L_{2} \cap R_{5}, & \Sigma_{3}^{-}=L_{1} \cap R_{5},
\end{array}
$$

with

$$
\begin{array}{ll}
R_{1}=\{-1<x<-1 / 2\}, & R_{2}=\{-1 / 2<x<0\}, \\
R_{3}=\{0<x<l-1 / 2\}, & R_{4}=\{l-1 / 2<x<l\}, \\
R_{5}=\{l<x<2 l\} . &
\end{array}
$$

The defect of $\varphi_{0}$ consists of

$$
\begin{gathered}
\Sigma_{i}^{ \pm} \quad i=1,2,3 \text { and } \\
\Gamma_{1}=\{y=0\} \cap R_{2}, \quad \Gamma_{2}=\{y=0\} \cap\left(R_{3} \cup R_{4}\right), \\
\Gamma^{+}=\left\{x^{2}+\left(y-\frac{1}{2}\right)^{2}=(l-y)^{2}, y>0\right\} \cap R_{4}, \\
\Gamma^{-}=\left\{x^{2}+\left(y+\frac{1}{2}\right)^{2}=(l+y)^{2}, y<0\right\} \cap R_{4} ;
\end{gathered}
$$

see Figure 3.1. The defect of $\varphi_{2}$ consists of $\Sigma_{i}^{ \pm}(i=1,2,3) S_{i}^{ \pm}(i=1,2)$ and

$$
C=\{x=0,|y|<1 / 2\} \text {, }
$$

with jump $j=2$ on $C$. 


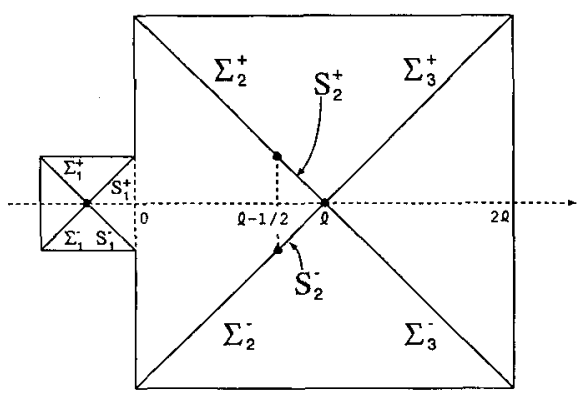

defects of $\varphi_{1}$

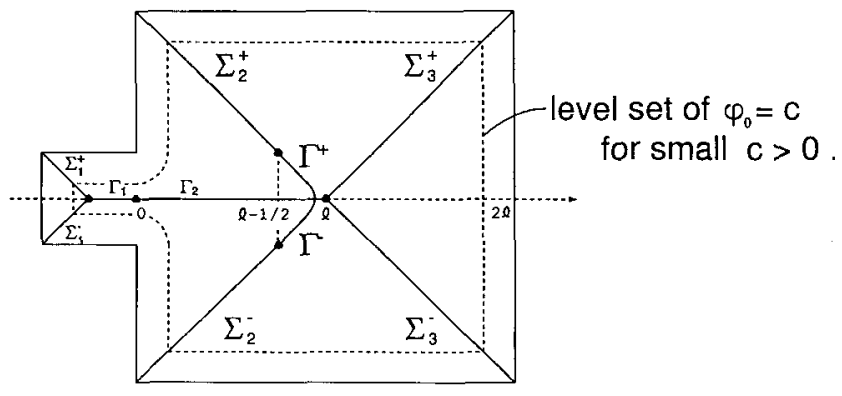

defects of $\varphi_{0}$

Figure 3.1.

\section{Computation of defect energy}

We shall estimate the difference $J^{\beta}\left(\varphi_{0}\right)-J^{\beta}\left(\varphi_{1}\right)$. By symmetry with respect to $y=$ 0 , we observe that

$$
\begin{aligned}
& J^{\beta}\left(\varphi_{0}\right)=2 \sum_{i=1}^{3} J^{\beta}\left(\varphi_{0}, \Sigma_{i}^{+}\right)+\sum_{i=1}^{2} J^{\beta}\left(\varphi_{0}, \Gamma_{i}\right)+2 J^{\beta}\left(\varphi_{0}, \Gamma^{+}\right), \\
& J^{\beta}\left(\varphi_{1}\right)=2 \sum_{i=1}^{3} J^{\beta}\left(\varphi_{1}, \Sigma_{i}^{+}\right)+2 \sum_{i=1}^{2} J^{\beta}\left(\varphi_{1}, S_{i}^{+}\right),
\end{aligned}
$$

where

$$
J^{\beta}(\varphi, B)=\int_{\Sigma \cap B} j^{\beta} d \mathscr{H}^{n-1} \quad \text { with } \quad j=\left|\nabla \varphi^{+}-\nabla \varphi^{-}\right| .
$$

Since jump $j$ is the same both for $\varphi_{0}$ and $\varphi_{1}$ on $\Sigma_{i}^{+}$, 


$$
J^{\beta}\left(\varphi_{0}, \Sigma_{i}^{+}\right)=J^{\beta}\left(\varphi_{1}, \Sigma_{i}^{+}\right), \quad i=1,2,3 .
$$

Thus

$$
J^{\beta}\left(\varphi_{0}\right)-J^{\beta}\left(\varphi_{1}\right)=\sum_{i=1}^{2} J^{\beta}\left(\varphi_{0}, \Gamma_{i}\right)+2 J^{\beta}\left(\varphi_{0}, \Gamma^{+}\right)-2 \sum_{i=1}^{2} J^{\beta}\left(\varphi_{1}, S_{i}^{+}\right)
$$

Proposition 3.1. (i) $J^{\beta}\left(\varphi_{0}, \Gamma_{1}\right)=2^{\beta-1}$.

(ii) $\lim _{l \rightarrow \infty} J^{\beta}\left(\varphi_{0}, \Gamma_{2}\right)=\int_{0}^{\infty} \frac{d x}{\left(x^{2}+1 / 4\right)^{\beta / 2}} \geqq \frac{1}{\beta-1} 2^{\beta-1}$ for $\beta>1 . \quad$ For $\quad \beta \leqq 1$, $\lim _{l \rightarrow \infty} J^{\beta}\left(\varphi_{0}, \Gamma_{2}\right)=\infty$.

(iii) $J^{\beta}\left(\varphi_{0}, \Gamma^{+}\right) \rightarrow 2^{(\beta-1) / 2}$ as $l \rightarrow \infty$.

(iv) $J^{\beta}\left(\varphi_{1}, S_{i}^{+}\right)=2^{(\beta-1) / 2}, i=1,2$.

Proof. (i) Clearly, $j=2$ on $\Gamma_{1}$. Since the length of $\Gamma_{1}$ is $1 / 2, J^{\beta}=2^{\beta-1}$.

(ii) Since the level curve of $\varphi_{0}$ intersecting $(x, 0)(0<x<l)$ is the circle centred $(0,1 / 2)$ for $y>0$, the normal component of $\nabla \varphi_{0}^{+}$equals

$$
\frac{1}{2} \frac{1}{\left(x^{2}+1 / 4\right)^{1 / 2}}
$$

Thus

$$
J^{\beta}\left(\varphi_{0}, \Gamma_{2}\right)=\int_{0}^{l} \frac{d x}{\left(x^{2}+1 / 4\right)^{\beta / 2}} \geqq \int_{0}^{l} \frac{d x}{(x+1 / 2)^{\beta}}=\left.\frac{1}{\beta-1}\left(x+\frac{1}{2}\right)^{1-\beta}\right|_{l} ^{0}
$$

and letting $l \rightarrow \infty$ completes the proof.

(iii) Recall that $\Gamma^{+}$is a set of points whose distance to $(0,1 / 2)$ equals the distance to the line $y=l$. The curve $\Gamma^{+}$is a parabola given by

$$
(2 l-1) y=-x^{2}+l^{2}-1 / 4
$$

The jump

$$
j(x)=2 \frac{1}{\left(y^{\prime}(x)^{2}+1\right)^{1 / 2}}
$$

and the length element equals $\left(y^{\prime}(x)^{2}+1\right)^{1 / 2} d x$. If $p$ is the largest zero of $y(x)$, i.e.

$$
p=\left(l^{2}-1 / 4\right)^{1 / 2},
$$

then

$$
J^{\beta}\left(\varphi_{0}, \Gamma^{+}\right)=\int_{l-1 / 2}^{p}\left(2 \frac{1}{\left(\left(y^{\prime}\right)^{2}+1\right)^{1 / 2}}\right)^{\beta}\left(\left(y^{\prime}\right)^{2}+1\right)^{1 / 2} d x
$$


Notice that

$$
\begin{aligned}
y^{\prime}(x)^{2}+1 & =\left(\frac{-2 x}{2 l-1}\right)^{2}+1, \quad l-1 / 2<x<l \\
& \rightarrow 2 \quad \text { as } l \rightarrow \infty, \\
p-(l-1 / 2)= & \left(l^{2}-1 / 4\right)^{1 / 2}-(l-1 / 2)=\frac{1}{2}+\frac{-2 / 4}{\left(l^{2}-1 / 4\right)^{1 / 2}+l} \\
\rightarrow \frac{1}{2} & \text { as } l \rightarrow \infty .
\end{aligned}
$$

We thus conclude that

$$
J^{\beta}\left(\varphi_{0}, \Gamma^{+}\right)=\int_{l-1}^{p} 2^{\beta}\left(\left(y^{\prime}\right)^{2}+1\right)^{(1-\beta) / 2} d x \rightarrow 2^{\beta} 2^{(1-\beta) / 2} \cdot \frac{1}{2}=2^{(\beta-1) / 2} \quad \text { as } l \rightarrow \infty .
$$

(iv) Since $j=\sqrt{2}$ and the length of $S_{i}^{+}$equals $1 / \sqrt{2}$, the result follows immediately.

Proposition 3.2. Let $\Omega$ be the domain $\Omega_{l}$ defined in the first subsection. Let $\varphi_{0}$ be the distance function of $\partial \Omega$ and let $\varphi_{1}$ and $\varphi_{2}$ be solutions of the eikonal equation defined in the second subsection.

(i) $\lim _{l \rightarrow \infty}\left(J^{\beta}\left(\varphi_{0}\right)-J^{\beta}\left(\varphi_{1}\right)\right)=\infty$ for $0<\beta \leqq 1$.

(ii) $\lim _{l \rightarrow \infty}\left(J^{\beta}\left(\varphi_{0}\right)-J^{\beta}\left(\varphi_{2}\right)\right)=\infty$ for $0<\beta \leqq 1$.

(iii) $\lim _{l \rightarrow \infty}\left(J^{\beta}\left(\varphi_{0}\right)-J^{\beta}\left(\varphi_{1}\right)\right)>0$ for all $\beta>0$.

(iv) $\lim _{l \rightarrow \infty}\left(J^{\beta}\left(\varphi_{0}\right)-J^{\beta}\left(\varphi_{2}\right)\right)>0$ for all $0<\beta<\beta_{0}$ with some $\beta_{0}>4 / 3$.

Proof. Applying Proposition 3.1 to the formula $J^{\beta}\left(\varphi_{0}\right)-J^{\beta}\left(\varphi_{1}\right)$ yields

$$
\begin{aligned}
T=\lim _{l \rightarrow \infty}\left(J^{\beta}\left(\varphi_{0}\right)-J^{\beta}\left(\varphi_{1}\right)\right)= & 2^{\beta-1}+\lim _{l \rightarrow \infty} J^{\beta}\left(\varphi_{0}, \Gamma_{2}\right)+2 \cdot 2^{(\beta-1) / 2} \\
& -2\left(2^{(\beta-1)}+2^{(\beta-1) / 2}\right) \\
= & 2^{\beta-1}-2 \cdot 2^{(\beta-1) / 2}+\lim _{l \rightarrow \infty} J^{\beta}\left(\varphi_{0}, \Gamma_{2}\right) .
\end{aligned}
$$

If $\beta \leqq 1$, then this formula yields (i). Note that

$$
J^{\beta}\left(\varphi_{2}\right)=J^{\beta}\left(\varphi_{1}\right)+J^{\beta}\left(\varphi_{2}, C\right) \text { with } C=\bar{D} \cap \bar{D}_{l} .
$$

Since $J^{\beta}\left(\varphi_{2}, C\right)=1 \cdot 2^{\beta}$, the proof of (ii) is now complete.

To show (iii), we may assume $\beta>1$ and use the estimate

$$
\lim _{l \rightarrow \infty} J^{\beta}\left(\varphi_{0}, \Gamma_{2}\right) \geqq \frac{1}{\beta-1} 2^{\beta-1},
$$

to get

$$
T \geqq 2 \cdot 2^{(\beta-1) / 2}(f(\beta)-1),
$$

with

$$
f(\beta)=2^{(\beta-3) / 2} \frac{\beta}{\beta-1} .
$$


An elementary calculation shows that $f$ takes the only minimum at $\beta=\beta_{1}$ over all $\beta>0$. The number $\beta_{1}$ is the solution of

$$
-\frac{1}{\beta_{1}-1}+\frac{1}{2}(\log 2) \beta_{1}=0, \quad \text { so that } \beta_{1} \geqq 1 \text {. }
$$

Since $\log 2>1 / 2,\left(\beta_{1}-1\right) \beta_{1} \leqq 1 / 4$ so that $\beta_{1}-1 \leqq 1 / 2$. Since $\beta_{1} \geqq 1$, we now obtain

$$
\begin{aligned}
f(\beta) \geqq f\left(\beta_{1}\right) & =2^{\left(\beta_{1}-3\right) / 2}\left(1+\frac{1}{\beta_{1}-1}\right) \\
& >2^{-1}(1+2) .
\end{aligned}
$$

We thus conclude, for sufficiently large $l$,

$$
J^{\beta}\left(\varphi_{0}\right)>J^{\beta}\left(\varphi_{1}\right)+2 \cdot 2^{(\beta-1) / 2} \cdot 1 / 2 .
$$

It remains to prove (iv). We may assume $\beta>1$. Notice that $J^{\beta}\left(\varphi_{2}, C\right)=2^{\beta}$ to get

$$
\begin{aligned}
T & \geqq \frac{\beta}{\beta-1} 2^{\beta-1}-2 \cdot 2^{(\beta-1) / 2}-2^{\beta} \\
& =2 \cdot 2^{(\beta-1)}\left(\left(\frac{1}{\beta-1}-1\right) 2^{(\beta-3) / 2}-1\right) .
\end{aligned}
$$

The right-hand side is positive if $\beta \leqq 4 / 3$.

THEOREM 3.3. Assume that $\Omega=\Omega_{l}$.

(i) $(\beta \leqq 1)$ For each $M>0$, there is a constant $l_{0}=l_{0}(\beta)$ such that, if $l>l_{0}(\beta)$, then

$$
J^{\beta}\left(\varphi_{0}\right) \geqq J^{\beta}\left(\varphi_{2}\right)+M \geqq J^{\beta}\left(\varphi_{1}\right)+M \text {. }
$$

Moreover, $\varphi_{0}$ minimises neither $I$ nor $J^{\beta}$ in $A_{+}, A$.

(ii) $(\beta>1)$ There is a constant $l_{1}=l_{1}(\beta)$ such that $\varphi_{0}$ does not minimise $J^{\beta}$ in $A$ for $l>l_{1}(\beta)$. Moreover, if $\beta \leqq 4 / 3$ (or $\beta<\beta_{0}$ ), then $\varphi_{0}$ does not minimise $J^{\beta}$ in $A_{+}$.

Proof. (i) The first inequality follows from Proposition 3.2(ii) and

$$
J^{\beta}\left(\varphi_{2}\right)=J^{\beta}\left(\varphi_{1}\right)+2^{\beta}
$$

for sufficiently large $l$. Since $\varphi_{0} \in A_{+} \subset A, \varphi_{1} \in A^{0}$ and $\varphi_{2} \in A_{+}^{0}$, we now observe that

$$
I\left(\varphi_{0}\right) \geqq J^{1}\left(\varphi_{0}\right)>I\left(\varphi_{2}\right)=J^{1}\left(\varphi_{2}\right)>I\left(\varphi_{1}\right)=J^{1}\left(\varphi_{1}\right),
$$

and

$$
J^{\beta}\left(\varphi_{0}\right)>J^{\beta}\left(\varphi_{2}\right)>J^{\beta}\left(\varphi_{1}\right) \text { for } \beta \leqq 1 .
$$

(ii) Since $\varphi_{0} \in A_{+}, \varphi_{1} \in A^{0}$ and $\varphi_{2} \in A_{+}^{0}$, Proposition 3.2 yields the desired conclusion.

\section{Acknowledgment}

The authors are grateful to Professor Robert Kohn for his interest and valuable comments on this paper. Much of this work was done while the second author was visiting the Japan-American Mathematics Institute of the Johns Hopkins University. 
Its hospitality is gratefully acknowledged, as is support from the Japan Society for the Promotion of Science. This work is partly supported by the Suhara Memorial Foundation and Yamada Science Foundation.

\section{References}

1 G. Alberti. Rank one property for derivatives of functions with bounded variation. Proc. Roy. Soc. Edinburgh Sect. A 123 (1993), 239-74.

2 P. Aviles and Y. Giga. A mathematical problem related to the physical theory of liquid crystal configurations. Proc. Centre Math. Anal. Austral. Nat. Univ. 12 (1987), 1-16.

3 P. Aviles and Y. Giga. Singularities and rank one properties of Hessian measures. Duke Math. J. 58 (1989), 441-67.

4 P. Aviles and Y. Giga. Variational integrals on mappings of bounded variation and their lower semicontinuity. Arch. Rational Mech. Anal. 115 (1991), 201-55.

5 F. H. Clarke. Optimization and Nonsmooth Analysis (New York: John Wiley, 1983).

6 M. G. Crandall, H. Ishii and P.-L. Lions. User's guide to viscosity solutions of second order partial differential equations. Bull. Amer. Math. Soc. 27 (1992), 1-67.

7 E. Giusti. Minimal Surfaces and Functions of Bounded Variations (Boston: Birkhauser, 1984).

8 R. Kohn and S. Muller. Relaxation and regularization of nonconvex variational problems. Rend. Semi. Mat. Fis. Univ. Milano 62 (1992), 89-113.

9 R. Kohn and S. Muller. Surface energy and microstructure in coherent phase transition. Comm. Pure Appl. Math. 47 (1994), 405-35.

10 P. L. Lions. Generalized Solutions of Hamilton-Jacobi Equations (Boston: Pitman Advanced Publ. Program, 1982).

11 M. Ortiz and G. Gioia. The morphology and folding patterns of buckling driven thin-film filters. J. Mech. Phy. Solids 42 (1994), 531-59.

12 J. Sethna and M. Kleman. Spheric domains in smectic liquid crystals. Phys. Rev. A. 26 (1982), 3037-40.

13 L. Simon. Lectures on Geometric Measure Theory. (Canberra: Proc. Centre Math. Anal. Austral. Nat. Univ, 3 1983).

(Issued 14 October 1996) 